\title{
A Modified Vivaldi Antenna for Improved Angular-Dependent Fidelity Property
}

\author{
Zhi Zeng, ${ }^{1}$ Ke Xu, ${ }^{2}$ Zhaohui Song, ${ }^{3}$ and Qinyu Zhang ${ }^{1}$ \\ ${ }^{1}$ Shenzhen Graduate School, Harbin Institute of Technology, Shenzhen 518055, China \\ ${ }^{2}$ Graduate School, Harbin Institute of Technology, Harbin 150001, China \\ ${ }^{3}$ Harbin Institute of Technology, Harbin 150001, China \\ Correspondence should be addressed to Qinyu Zhang; zqy@hit.edu.cn
}

Received 23 December 2012; Revised 10 April 2013; Accepted 4 May 2013

Academic Editor: Renato Cicchetti

Copyright (c) 2013 Zhi Zeng et al. This is an open access article distributed under the Creative Commons Attribution License, which permits unrestricted use, distribution, and reproduction in any medium, provided the original work is properly cited.

\begin{abstract}
The analysis, design, and realization of a modified Vivaldi antenna optimized for time domain fidelity factor in the half-space located in the direction of the antenna main beam are presented. The proposed antenna shows improved angular-dependent fidelity property, with respect to the signal transmitted in the main beam direction. A substantial increase in the fidelity factor is achieved by utilizing spatial filter effect introduced by adding two dielectric slabs parallel to the antenna substrate. By choosing optimal dimensions and location of the slabs, the signal waveforms in the mentioned half-space are equalized so as to improve the quality of the radiated signal waveform in the main beam direction. As a result, the fidelity property in the half-space is improved greatly. The simulated and measured fidelity factor in the angular operational region is studied and compared with experimental measurements. The ranges with the fidelity factor better than the value of 0.9 are improved by $95 \%$ in $\mathrm{H}$-plane and by $14 \%$ in E-plane, respectively.
\end{abstract}

\section{Introduction}

Since Federal Communication Commission (FCC) of USA opened up the frequency range $3.1-10.6 \mathrm{GHz}$ for ultrawideband (UWB) communication in 2002, UWB has attracted a lot of attention in the wireless communications field from both academia and industries [1]. UWB system has many advantages, such as high data rate, high spatial resolution, low-cost transceiver, low transmit power, and low interference, which are quite suited to short-range high-rate communication, real-time localization, and see-through-thewall and ground penetrating radar $[2,3]$. There have been lots of investigations about these UWB applications; some of them have already been commercially used.

UWB antenna is one of the key parts in UWB system. A well-designed UWB antenna should have a wide bandwidth, a stable gain pattern that ensures a flat magnitude of the transfer function, and a linear phase response, characteristics useful to minimize the distortion of the transmitted/received pulses [4]. In [5], a nondirective UWB printed antenna for wireless applications, characterized by a low group delay, excellent integration capability with the active/passive components forming the receiver/transmitter front end, and good radiative performances when arranged in arrays, has been presented. In [6], an UWB antenna that rejects extremely sharply the two narrow and closely spaced US WLAN 802.11a bands is presented, while in [7], a compact, low dispersive UWB antenna with sectorial radiation pattern and high front to back ratio has been proposed.

Vivaldi antenna is a kind of tapered slot UWB antenna. The first tapered slot antenna was presented by Lewis et al. in 1974 [8] and named Vivaldi antenna by Gibson in 1979 [9]. Vivaldi antennas are widely used in UWB system for its wide bandwidth, high directivity, low cross-polarization, and easy fabrication. During the past decades, many investigators studied Vivaldi antennas. The miniaturization and the bandwidth performances are key requirements in the design of modern wideband antennas. In this context, Hood presented a compact Vivaldi antenna fabricated with a $39.4 \times 34.6 \mathrm{~mm}$ substrate, which operates in the frequency band 3.3-10.6 GHz [10]. In [11], a slot loaded Vivaldi antenna with bandwidth over $25: 1$ is presented. In addition, the pulse 


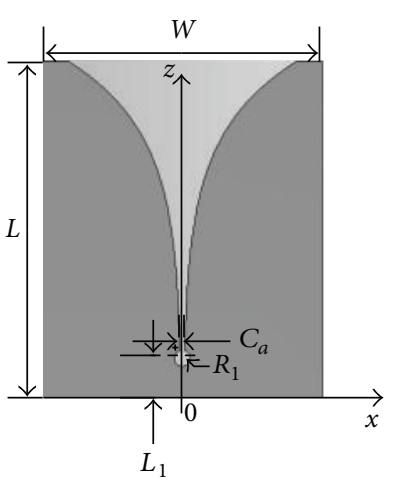

(a)

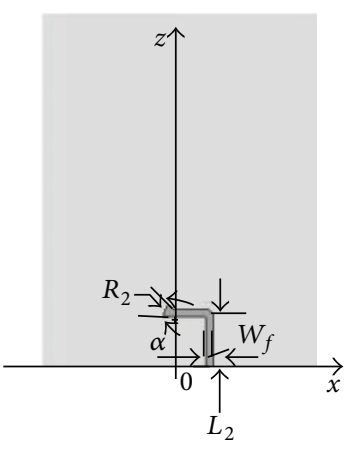

(b)

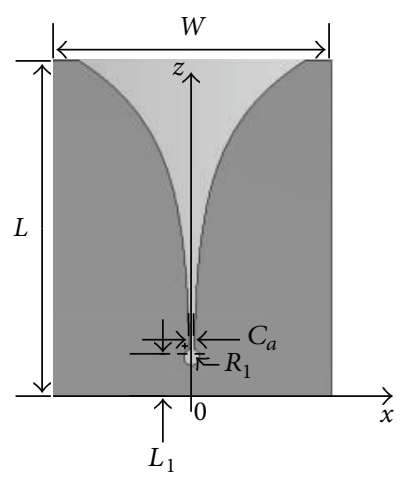

(c)

FIGURE 1: Geometry of the original Vivaldi antenna: (a) top view, (b) central layer (feed), and (c) bottom view.

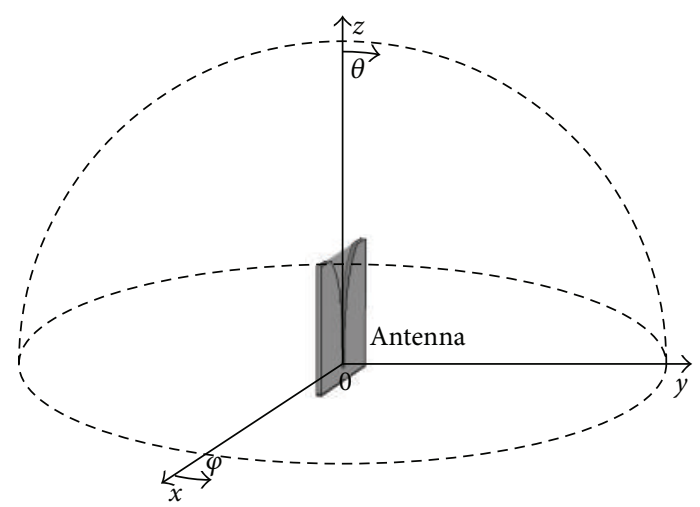

FIGURE 2: Vivaldi antenna (the reference frame is indicated in the figure).

radiation characteristics are quite important for antennas used in the impulse UWB system. In [12], the authors studied the transient distortion, reflection coefficient, and crosspolarization level of Vivaldi antenna. Vivaldi antenna is quite fitting for UWB antenna array due to its wide bandwidth, high directivity, low cross-polarization, and low profile. In the past decades, especially after the computer is getting powerful enough to analyze antenna arrays, many investigations were carried out to improve the performance of Vivaldi antenna arrays. As of this time, no competing array technology can match the wide bandwidth and wide scanning

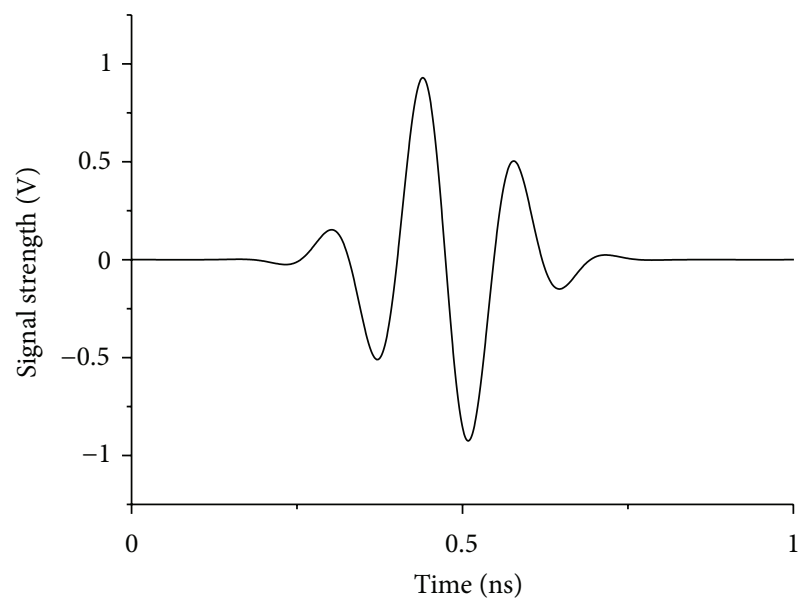

FIgURE 3: Excitation signal.

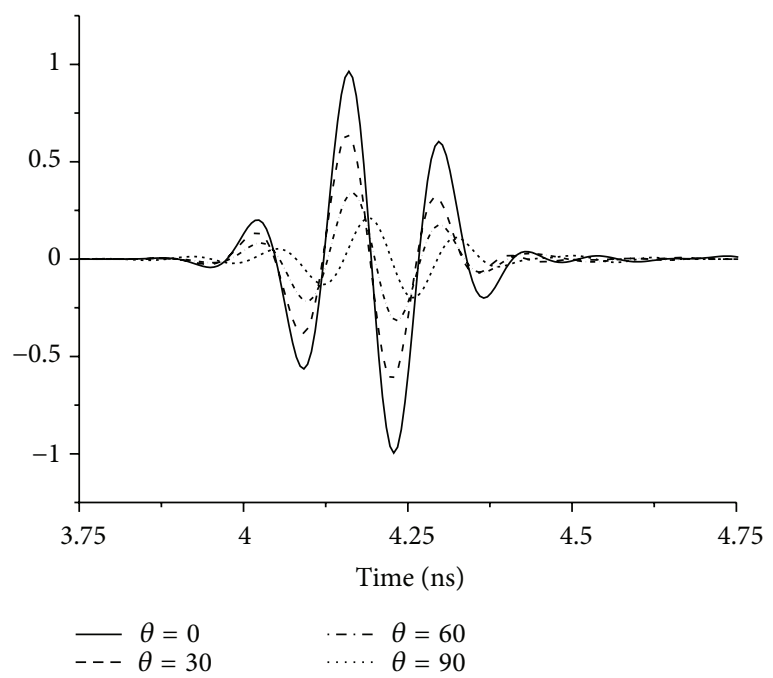

FIGURE 4: Normalized radiation signal waveform in E-plane $(\varphi=$ $\left.0^{\circ}\right)$.

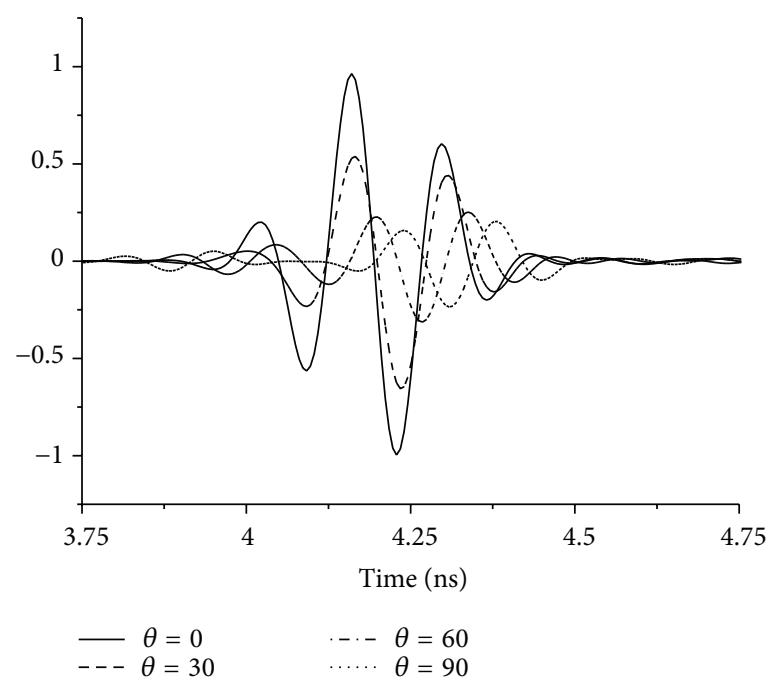

FIGURE 5: Normalized radiation signal waveform in $\mathrm{H}$-plane $(\varphi=$ $\left.90^{\circ}\right)$. 
impedance performance of Vivaldi antenna arrays, which are widely used in modern electronic warfare system and radar system $[13,14]$.

It is quite important for an antenna array element to keep good performance in a wide spatial region while the main beam is scanning. In practice, all the antennas radiate different signals in different spatial directions. So it is quite important to study the correlation between the signal radiated and spatial directions, both in frequency and in time domains. In [15], the angular distortion of the signal radiated with respect to that emitted in the main beam direction of UWB antennas has been investigated. The correlation properties of the pulse signals are determined by the so-called fidelity factor. It can be quantified through the analysis of the correlation between the signal in an arbitrary angular direction and the signal in the main beam direction. Usually, a fidelity factor which is greater than the value of 0.9 could be considered to be acceptable. In [16], Quintero et al. introduced system fidelity factor (SFF) to compare UWB antennas. In [3], the half-spherical fidelity factor pattern measurement results of Bowtie and Vivaldi antenna are presented. The fidelity factor pattern describes how does the signal vary in the different angular directions with respect to the signal in the main beam direction. In [17], Pancera and Wiesbeck introduced an optimization method based on the fidelity factor criterion to improve the radiation properties of Bowtie antennas for medical applications.

In this paper, a method to improve the fidelity factor of Vivaldi antenna in spatial range is presented. The original Vivaldi antenna operates in the frequency band 3.1-10.6 GHz. The modified antenna has two dielectric slabs that are parallel to the antenna substrate. These dielectric slabs are located at a distance $d$ from the upper and bottom faces of the antenna. The dielectric slabs act as a spatial filter to reform the signal waveform. Using the GA algorithm, the parameters of the dielectric slabs have been optimized producing an increment of the fidelity factor in a larger angular range. The simulations of both original antenna and improved antenna are performed using the commercial electromagnetic simulation software CST MWS. The available ranges with the fidelity factor greater than the value of $0.9 \mathrm{in} \mathrm{H}$-plane and in E-plane are improved by $95 \%$ and $14 \%$, respectively. The prototypes of both original antenna and improved antenna were fabricated and measured. The numerical results concerning the antenna parameters are found to be in good agreement with the experimental measurements.

\section{Improvement of the Vivaldi Antenna Fidelity Factor}

2.1. Original Vivaldi Antenna. Vivaldi antenna is one kind of classic end-fired directive travelling wave antennas, which has a tapered slot characterized by a bell-shaped exponential on it. The slot curve on the substrate board becomes wider gradually from the narrow end to the wide end, and it radiates the electromagnetic waves using the corresponding part of the slot as constant electric size at the relevant frequency. So theoretically, Vivaldi antenna has an infinite wide frequency

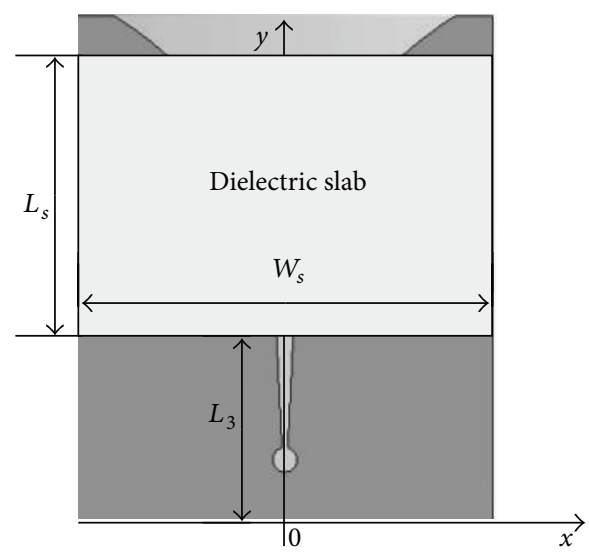

(a)

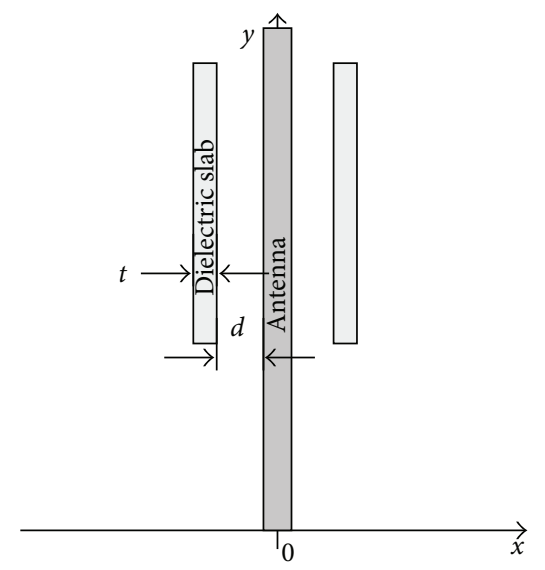

(b)

FIGURE 6: Geometry of the improved Vivaldi antenna: (a) top view and (b) lateral view.

band [5]. In practice, Vivaldi antenna has a band width better than $10: 1$ and has the features of low cross-polarization and highly directive radiation patterns over the whole frequency band. In this paper, a Vivaldi antenna operating in the frequency band $3.1-10.6 \mathrm{GHz}$ is investigated. The antenna is fabricated on a two-layer substrate with dielectric constant of 3.5 , size of $85 \mathrm{~mm} * 70 \mathrm{~mm}$, and each layer thickness of $0.8 \mathrm{~mm}$. The geometry of the original Vivaldi antenna is shown in Figure 1, with the $x z$-plane and $y z$-plane referred to as the E-plane and H-plane, respectively. The reference frame adopted to express the field quantities is shown in Figure 2. The tapers of the antennas are defined as

$$
x= \pm c_{a} \cdot \exp \left[c_{b}(y-10)\right] .
$$

The values of the antenna geometrical parameters are shown in Table 1.

A commercial Finite Integration Technique (FIT) electromagnetic simulation software CST MWS is used to analyze the radiative performances of the Vivaldi antenna. The antenna is excited by a UWB signal in the frequency band 3.110.6 GHz, which is a Gaussian pulse in the frequency band 0$3.75 \mathrm{GHz}$ modulated by a cosine wave carrier at the frequency 


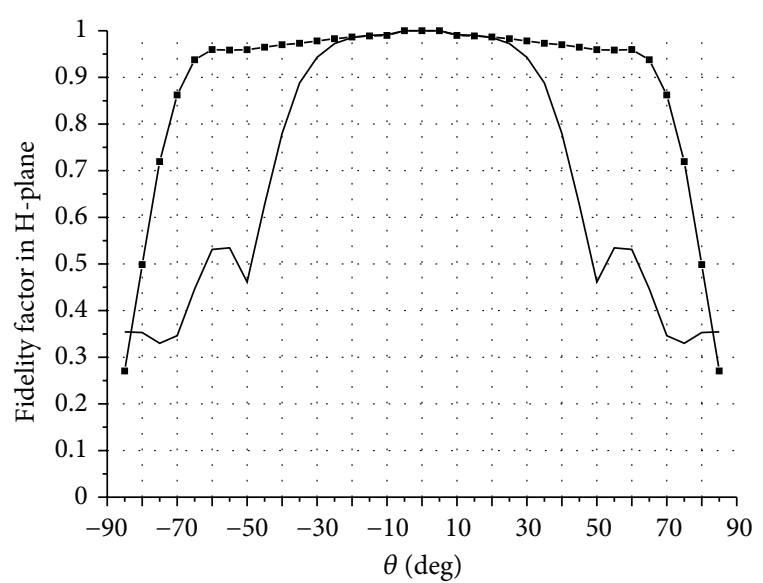

$\rightarrow$ Improved antenna

— Original antenna

(a)

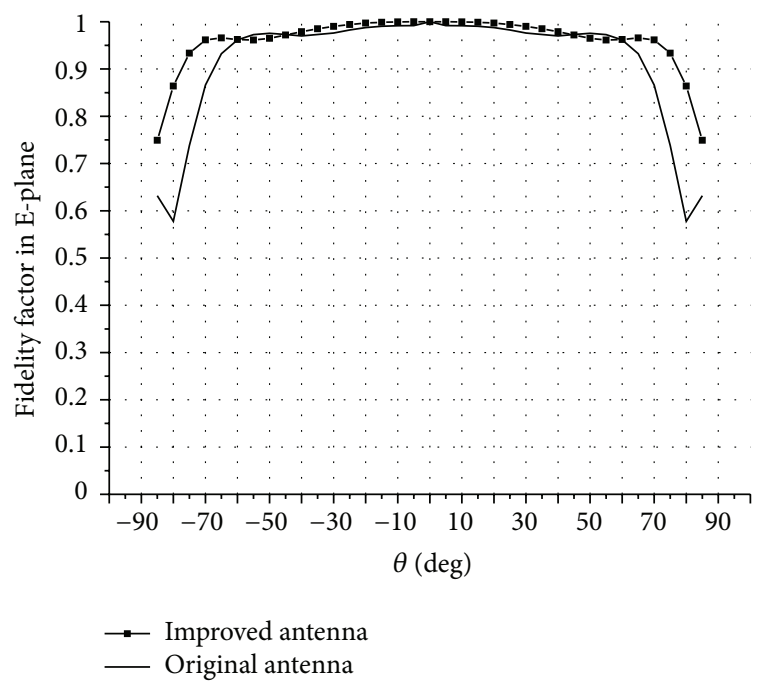

(b)

FIGURE 7: (a) Simulation results of fidelity factor in H-plane $(\varphi=$ $\left.90^{\circ}\right)$ and (b) simulation results of fidelity factor in E-plane $\left(\varphi=0^{\circ}\right)$.

6.85 GHz. The waveform of excitation signal is shown in Figure 3. A set of probes have been placed at a distance of $1000 \mathrm{~mm}$ from the antenna on a plane around the antenna and spaced by 5 degrees to detect the time-domain signal radiated by the antenna. Since the Vivaldi antenna has quite good cross-polar property, only the copolar radiation signals are considered. The normalized signal waveforms at the main beam direction and other directions computed in the E- and $\mathrm{H}$-planes are shown in Figures 4 and 5, respectively.

The fidelity factor between the signal at the main beam direction $S_{1}(t)$ and the signal in an arbitrary angular direction $S_{2}(t)$ is defined as the normalized cross-correlation between them and can be calculated by [3]

$$
\text { fidelity }=\max _{\tau} \frac{\int_{-\infty}^{+\infty} S_{1}(t-\tau) S_{2}(t) d t}{\sqrt{\int_{-\infty}^{+\infty}\left\|S_{1}(t)\right\|^{2} d t \int_{-\infty}^{+\infty}\left\|S_{1}(t)\right\|^{2} d t}} .
$$

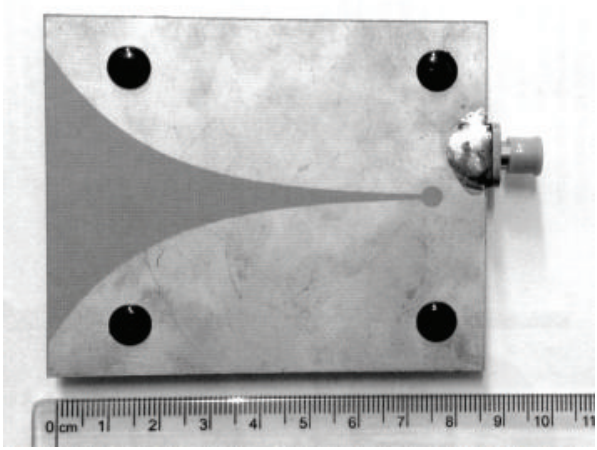

(a)

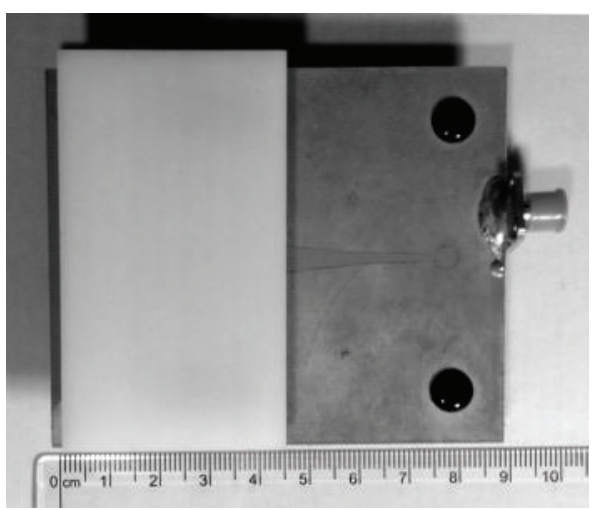

(b)

FIgURE 8: (a) Picture of the original antenna and (b) picture of the improved antenna.

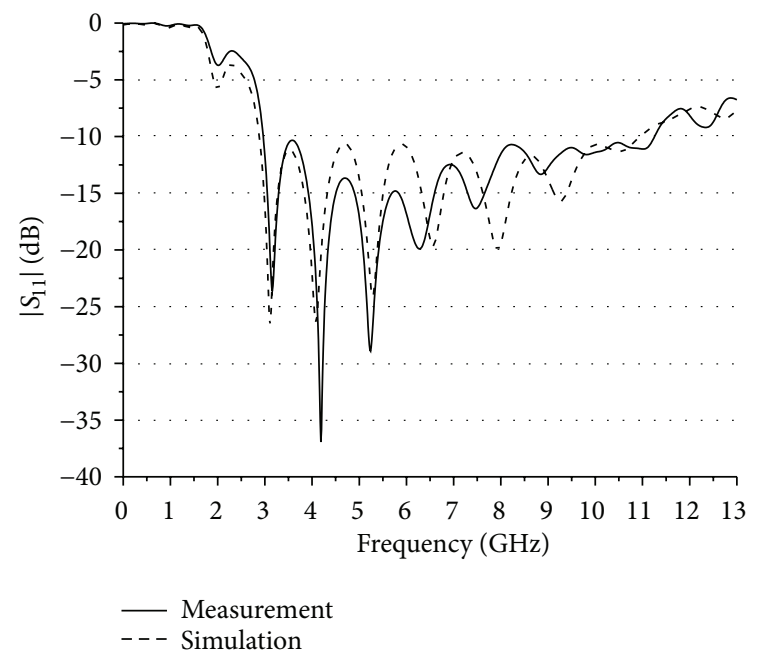

FIGURE 9: $\left|S_{11}\right|$ of improved antenna.

The fidelity factor results computed in E- and H-planes are listed in Table 2.

From Table 2, it appears that the fidelity factor in H-plane degrades quickly as the angle between the antenna's main beam and the observation point is larger than 35 degrees. 
TABLE 1: Geometrical parameters of the original Vivaldi antenna.

\begin{tabular}{lcc}
\hline Parameter & Value & Unit \\
\hline$W$ & 70 & $\mathrm{~mm}$ \\
$L$ & 85 & $\mathrm{~mm}$ \\
$L_{1}$ & 10 & $\mathrm{~mm}$ \\
$L_{2}$ & 12.25 & $\mathrm{~mm}$ \\
$W_{f}$ & 0.85 & $\mathrm{~mm}$ \\
$R_{1}$ & 2.07 & $\mathrm{~mm}$ \\
$R_{2}$ & 3.3 & $\mathrm{~mm}$ \\
$\alpha$ & 46 & $\circ$ \\
$C_{a}$ & 0.4440 & \\
$C_{b}$ & 0.0559 & \\
\hline
\end{tabular}

TABLE 2: Computed fidelity factor of the original antenna in H-plane and E-plane.

\begin{tabular}{lccc}
\hline \multicolumn{2}{c}{ H-plane $(\varphi=90)$} & \multicolumn{2}{c}{ E-plane $(\varphi=0)$} \\
$\theta$ (degree) & Fidelity factor & $\theta($ degree $)$ & Fidelity factor \\
\hline 0 & 1 & 0 & 1 \\
5 & 0.99998 & 5 & 0.99182 \\
10 & 0.99157 & 10 & 0.99154 \\
15 & 0.99012 & 15 & 0.99040 \\
20 & 0.98533 & 20 & 0.98758 \\
25 & 0.97260 & 25 & 0.98263 \\
30 & 0.94303 & 30 & 0.97626 \\
35 & 0.88816 & 35 & 0.97320 \\
40 & 0.77987 & 40 & 0.97004 \\
45 & 0.62700 & 45 & 0.97318 \\
50 & 0.46141 & 50 & 0.97558 \\
55 & 0.53453 & 55 & 0.97288 \\
60 & 0.53101 & 60 & 0.96154 \\
65 & 0.44638 & 65 & 0.93226 \\
70 & 0.34619 & 70 & 0.86615 \\
75 & 0.33003 & 75 & 0.73830 \\
80 & 0.35294 & 80 & 0.57766 \\
85 & 0.35444 & 85 & 0.63184 \\
\hline
\end{tabular}

And this distortion will cause poor performance degradation while the beam is scanning out of this range.

To improve the signal fidelity factor in a larger angular region, two dielectric slabs with relative dielectric constant $\varepsilon_{r}$ and thickness $t$, which parallel the antenna substrate, are added to the original Vivaldi antenna. The distance between the slabs and antenna substrate is $d$. The structure of the proposed antenna is shown in Figure 6.

The dielectric slabs play a role as a spatial filter, which have the angular-dependant effect on the incident wave. So, by proper tuning of $\varepsilon_{r}, t$, and $d$, the quality of the radiated wave can be restored. This effect can be used to optimize the antenna fidelity factor in the angular operational region. Besides the parameters above, the length of the slabs $L_{s}$ and the position of the slabs along the $y$-axis $L_{3}$ also can be considered as optimization parameters because they decide the angular range covered by the slabs.

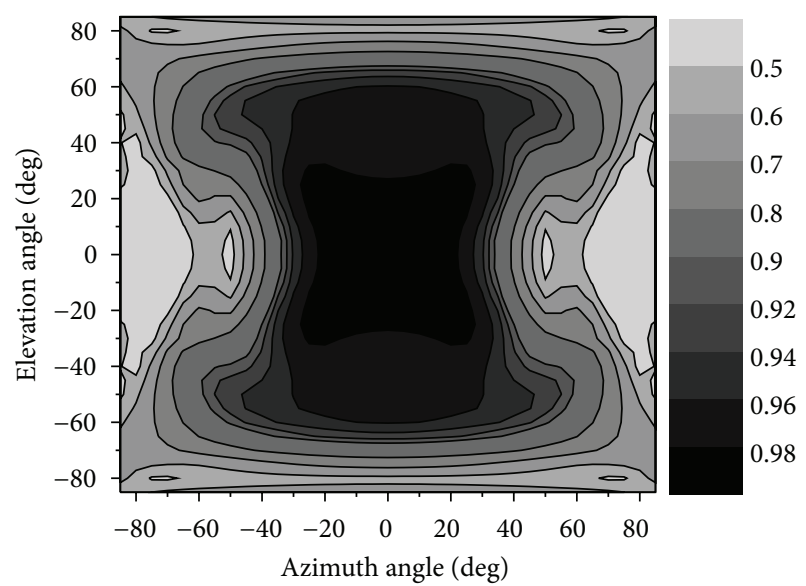

(a)

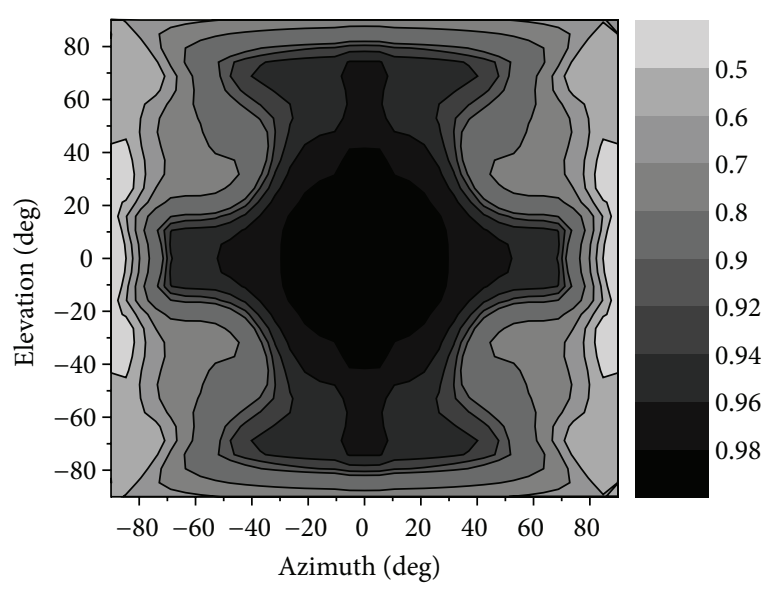

(b)

FIGURE 10: (a) Fidelity factor test results of the original antenna and (b) fidelity factor test results of the improved antenna.

\section{Antenna Simulation and Measurement}

The polyformaldehyde, having a relative dielectric constant $\varepsilon_{r}=3.8$, is chosen for the realization of the two dielectric slabs thanks to its intrinsic characteristics, consisting in the easy machining and good temperature characteristics. The structure of the Vivaldi antenna is optimized with the goal of the better fidelity factor in H-plane and in E-plane using Genetic Algorithms optimizer in CST MWS. The criterion of the fidelity factor is set to 0.9 . The optimal parameters of the dielectric slabs are listed in Table 3.

The fidelity factor of improved antenna computed in Eplane and H-plane is listed in Table 4 and compared with the results of the original antenna as shown in Figure 7.

The fidelity factor in H-plane has been improved significantly. The available range of the original antenna in $\mathrm{H}$-plane is 70 degrees, and the improved antenna has an available range wider than 135 degrees, which has been improved by about $97 \%$. Besides that, the available range in E-plane has been improved from 136 degrees to 156 degrees, which is not such remarkable as that in $\mathrm{H}$-plane. 


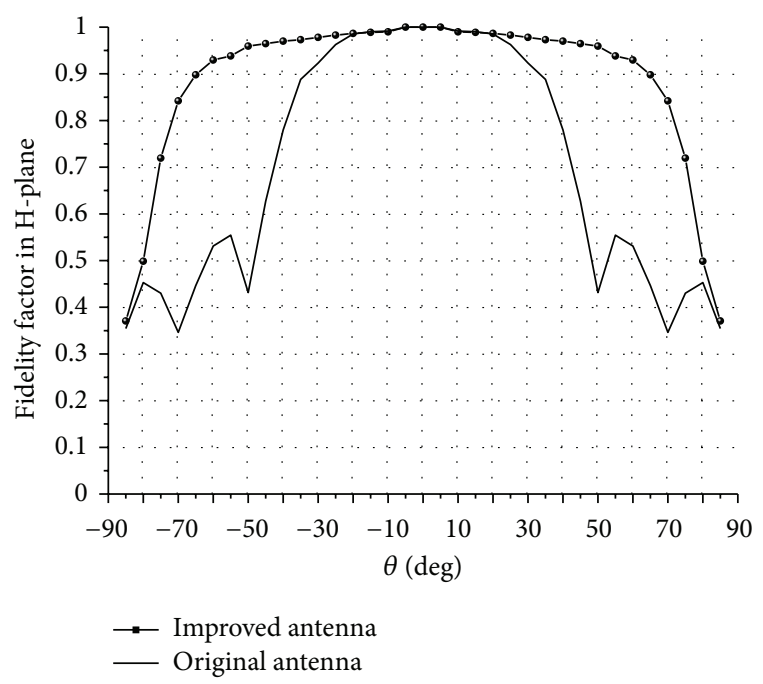

(a)

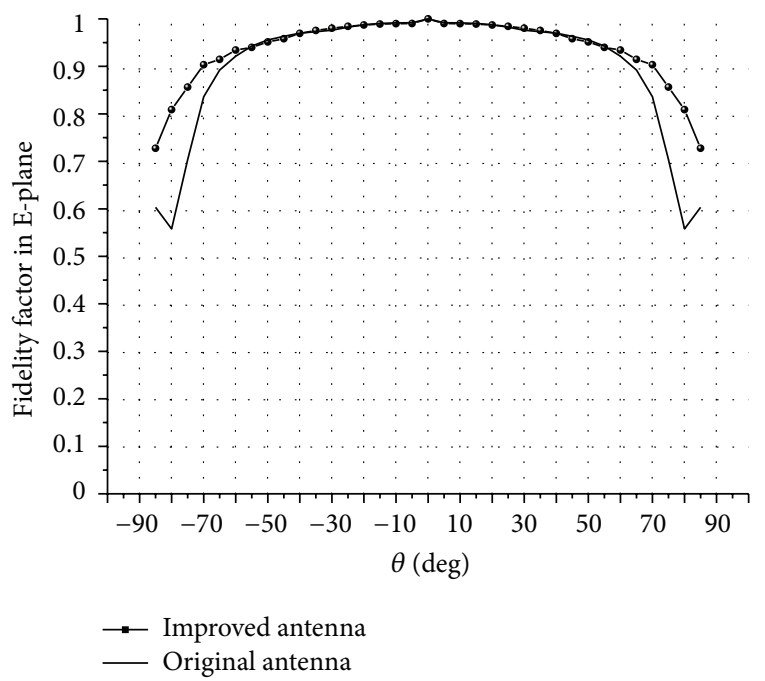

(b)

FIGURE 11: (a) Measurement results of fidelity factor in $\mathrm{H}$-plane and (b) measurement results of fidelity factor in E-plane.

TABLE 3: The geometrical parameters of the dielectric slabs.

\begin{tabular}{lcc}
\hline Parameter & Value & Unit \\
\hline$t$ & 4.4 & $\mathrm{~mm}$ \\
$W_{s}$ & 40 & $\mathrm{~mm}$ \\
$L_{3}$ & 43 & $\mathrm{~mm}$ \\
$d$ & 9.15 & $\mathrm{~mm}$ \\
$L_{s}$ & 70 & $\mathrm{~mm}$ \\
\hline
\end{tabular}

The prototypes of the two considered antennas are shown in Figure 8, while the numerical and measurement results concerning the frequency behavior of the parameter $\left|S_{11}\right|$ of the new antenna are reported in Figure 9.

The measurement of fidelity factor was carried out in an enclosed anechoic chamber. The measured antenna is installed on a rotating platform. A wideband probe is used
TABLE 4: Fidelity factor of the improved antenna computed in Eplane and H-plane.

\begin{tabular}{lccc}
\hline \multicolumn{2}{c}{ H-plane $(\varphi=90)$} & \multicolumn{2}{c}{ E-plane $(\varphi=0)$} \\
$\theta$ (degree) & Fidelity factor & $\theta($ degree $)$ & Fidelity factor \\
\hline 0 & 1 & 0 & 1 \\
5 & 0.99995 & 5 & 0.99995 \\
10 & 0.98976 & 10 & 0.99973 \\
15 & 0.98881 & 15 & 0.99888 \\
20 & 0.98655 & 20 & 0.99709 \\
25 & 0.98285 & 25 & 0.99421 \\
30 & 0.97808 & 30 & 0.99023 \\
35 & 0.97300 & 35 & 0.98521 \\
40 & 0.97008 & 40 & 0.97907 \\
45 & 0.96478 & 45 & 0.97186 \\
50 & 0.95924 & 50 & 0.96504 \\
55 & 0.95839 & 55 & 0.96137 \\
60 & 0.95966 & 60 & 0.96244 \\
65 & 0.93783 & 65 & 0.96566 \\
70 & 0.86216 & 70 & 0.96156 \\
75 & 0.71946 & 75 & 0.93351 \\
80 & 0.49866 & 80 & 0.86404 \\
85 & 0.27054 & 85 & 0.74927 \\
\hline
\end{tabular}

to receive the transient signal radiated by the antenna under test when changing the azimuth and the elevation angle of the antenna. The fidelity factors, calculated in the upper halfplane are plotted in Figure 10. The measurement results of the fidelity factor in E-plane and H-plane are shown in Figure 11.

The original Vivaldi antenna shows its good fidelity factor characteristics in a side-ways $\mathrm{H}$-shape area with azimuth angle scanning less than 35 degrees in $\mathrm{H}$-plane and elevation angle less than 65 degrees in E-plane, respectively. The improved Vivaldi antenna shows its good fidelity factor characteristics in a cross-shape area with azimuth angle scanning better than 65 degrees in H-plane and elevation angle better than 70 degrees in E-plane, respectively, which is extended in horizontal direction significantly.

\section{Conclusion}

In this paper, a new method to improve the fidelity factor of a planar Vivaldi UWB antenna has been proposed. Two dielectric slabs, which play the role of a spatial filter suitable to restore the signal waveform in time domain, are added to the original Vivaldi antenna. By tuning the dimensions and the relative position of the dielectric slabs, the fidelity factor in the half space is optimized. The angular available range in $\mathrm{H}$-plane with the fidelity factor greater than the value of 0.9 has been improved by $95 \%$ and the available angular range in E-plane has been improved by $14 \%$.

\section{Acknowledgments}

The authors would like to express their sincere gratitude to CST Ltd., Germany, for providing a free package of CST MWS 
software. This work was supported in part by the key program of the National Natural Science Foundation of China under Grant 61032003 and the National Basic Research Program of China under Grant 2009CB320402.

\section{References}

[1] Y. Q. Zhang, Y. X. Guo, and M. S. Leong, "A novel multilayer UWB antenna on LTCC," IEEE Transactions on Antennas and Propagation, vol. 58, no. 9, pp. 3013-3019, 2010.

[2] T. A. Vu, M. Z. Dooghabadi, S. Sudalaiyandi et al., "UWB Vivaldi antenna for impulse radio beamforming," in Proceedings of the 27th Norchip Conference, pp. 1-5, Trondeim, Norway, November 2009.

[3] E. Pancera, T. Zwick, and W. Wiesbeck, "Spherical fidelity patterns of UWB antennas," IEEE Transactions on Antennas and Propagation, vol. 59, no. 6, pp. 2111-2119, 2011.

[4] F. Gross, Frontiers in Antennas: Next Generation Design \& Engineering, McGraw-Hill, 2011.

[5] G. Cappelletti, D. Caratelli, R. Cicchetti, and M. Simeoni, "A low profile printed drop-shaped dipole antenna for wideband wireless applications," IEEE Transactions on Antennas and Propagation, vol. 59, no. 10, pp. 3526-3535, 2011.

[6] A. A. Gheethan and D. E. Anagnostou, "Dual band-reject UWB antenna with sharp rejection of narrow and closely-spaced bands," IEEE Transactions on Antennas and Propagation, vol. 60, no. 4, pp. 2071-2076, 2012.

[7] L. Desrumaux, A. Godard, M. Lalande, V. Bertrand, J. Andrieu, and B. Jecko, "An original antenna for transient high power UWB arrays: the Shark antenna," IEEE Transactions on Antennas and Propagation, vol. 58, no. 8, pp. 2515-2522, 2010.

[8] L. R. Lewis, M. Fassett, and J. Hunt, "A broadband stripline array element," in Proceedings of the IEEE Antennas and Propagation Symposium Digest, pp. 335-337, Atlanta, Ga, USA, 1974.

[9] Y. Li and A. Chen, "Design and application of Vivaldi antenna array," in Proceedings of the 8th International Symposium on Antennas, Propagation and EM Theory (ISAPE '08), pp. 267-270, Kunming, China, November 2008.

[10] A. Z. Hood, T. Karacolak, and E. Topsakal, "A small antipodal vivaldi antenna for ultrawide-band applications," IEEE Antennas and Wireless Propagation Letters, vol. 7, pp. 656-660, 2008.

[11] J. Bai, S. Shi, and D. W. Prather, "Ultra-wideband slotloaded antipodal Vivaldi antenna array," in Proceedings of the IEEE International Symposium on Antennas and Propagation (APSURSI '11), pp. 79-81, Spokane, Wash, USA, 2011.

[12] J. Zhang, J. Wang, and W. Hu, "Analysis of UWB signal distortion in transmitting/receiving antenna systems," in Proceedings of the 9th International Symposium on Antennas Propagation and EM Theory (ISAPE '10), pp. 163-166, Guangzhou, China, December 2010.

[13] K. Trott, B. Cummings, R. Cavener, M. Deluca, J. Biondi, and T. Sikina, "Wideband phased array radiator," in Proceedings of the IEEE International Symposium on Phased Array Systems \& Technology, pp. 383-386, Boston, Mass, USA, October 2003.

[14] D. Carsenat and C. Decroze, "UWB antennas beamforming using passive time-reversal device," IEEE Antennas and Wireless Propagation Letters, vol. 11, pp. 779-782, 2012.

[15] M. A. Elmansouri and D. S. Filipovic, "Pulse distortion and mitigation thereof in spiral antenna-based UWB communication systems," IEEE Antennas and Wireless Propagation Letters, vol. 59, no. 10, pp. 3863-3871, 2011.
[16] G. Quintero, J. F. Zurcher, and A. K. Skrivervik, "System fidelity factor: a new method for comparing UWB antennas," IEEE Transactions on Antennas and Propagation, vol. 59, no. 7, pp. 2502-2512, 2011.

[17] E. Pancera and W. Wiesbeck, "Fidelity based optimization of UWB antenna-radiation for medical applications," in Proceedings of the IEEE International Symposium on Antennas and Propagation (APSURSI '11), p. 2411, Spokane, Wash, USA, July 2011. 

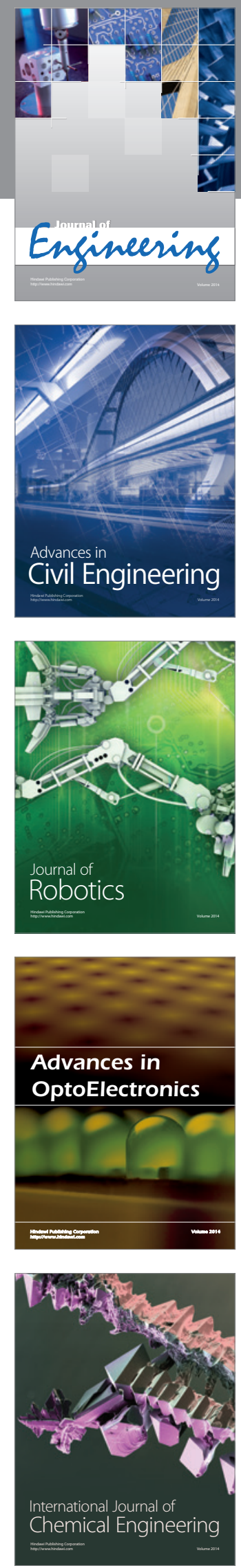

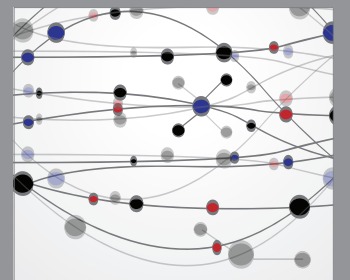

The Scientific World Journal
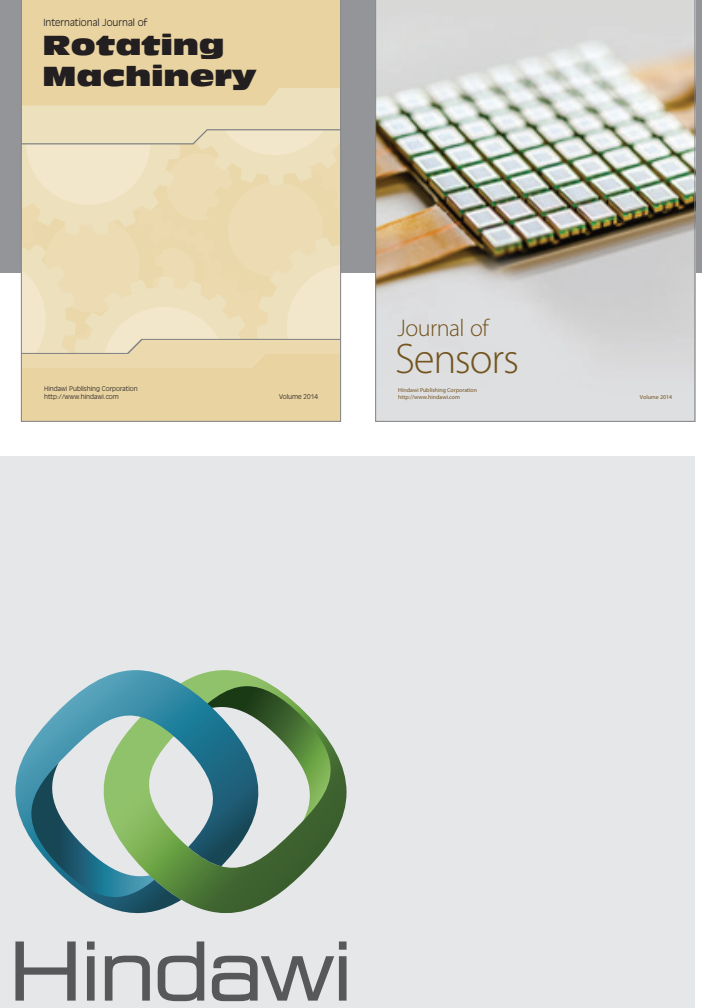

Submit your manuscripts at http://www.hindawi.com
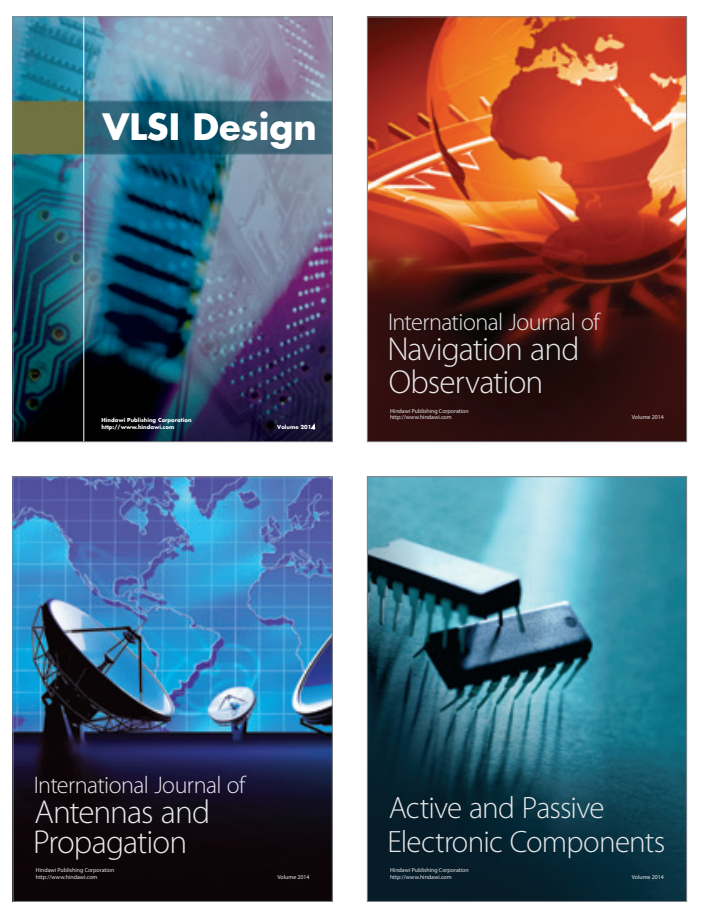
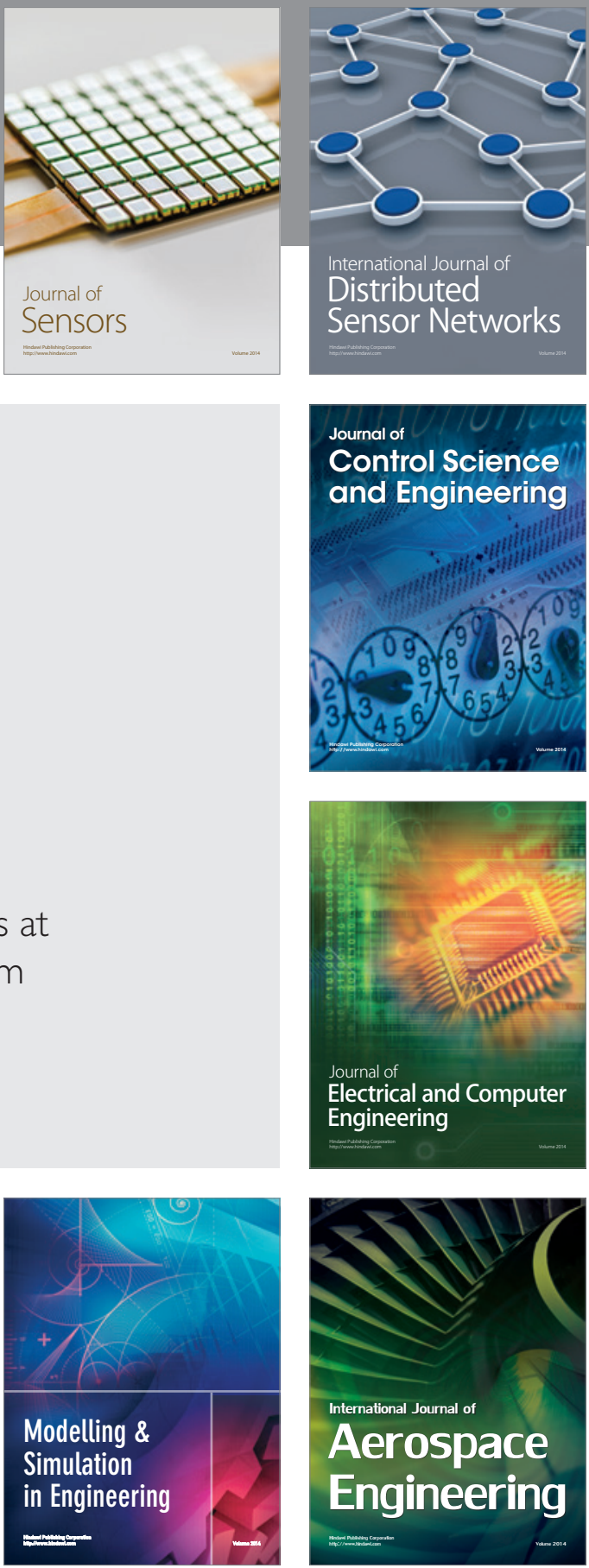

Journal of

Control Science

and Engineering
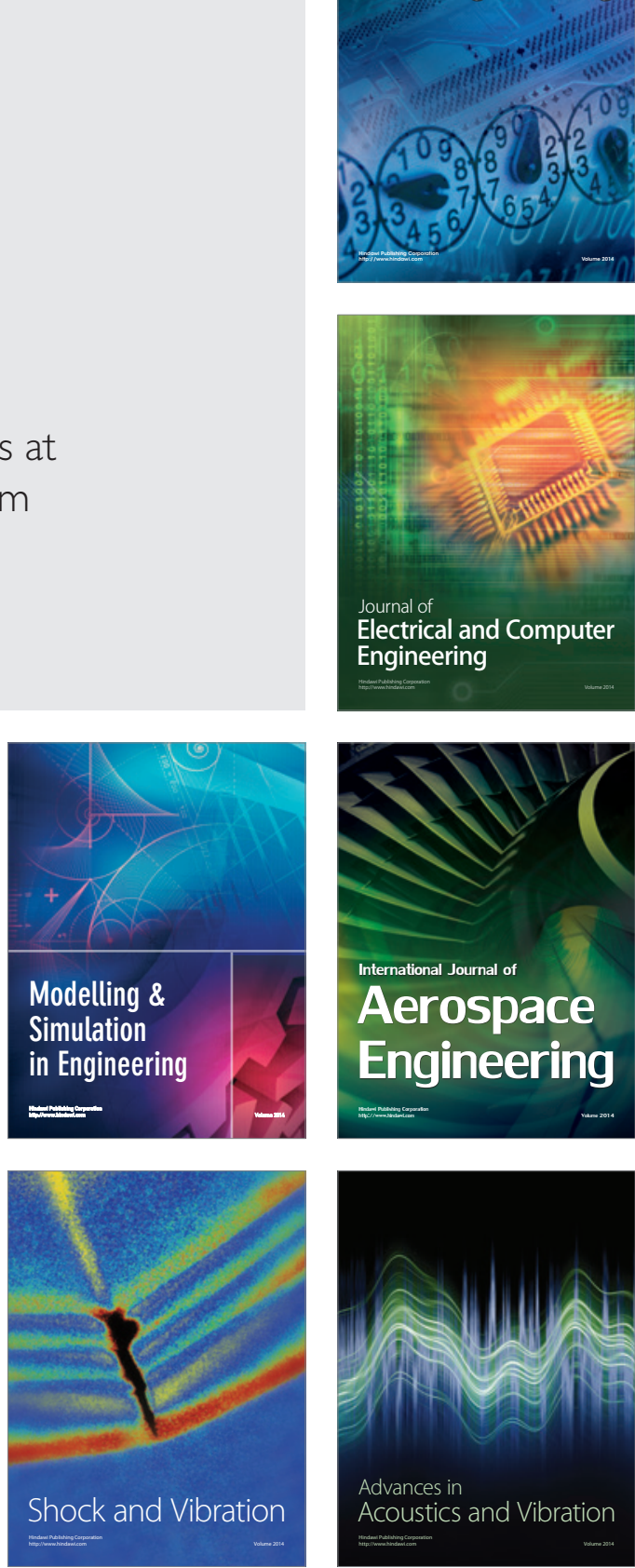